\title{
Composición nutricional de tres pastos colectados en el estado de Morelos, México
}

\section{Nutritional composition of three grasses from the state of Morelos, Mexico}

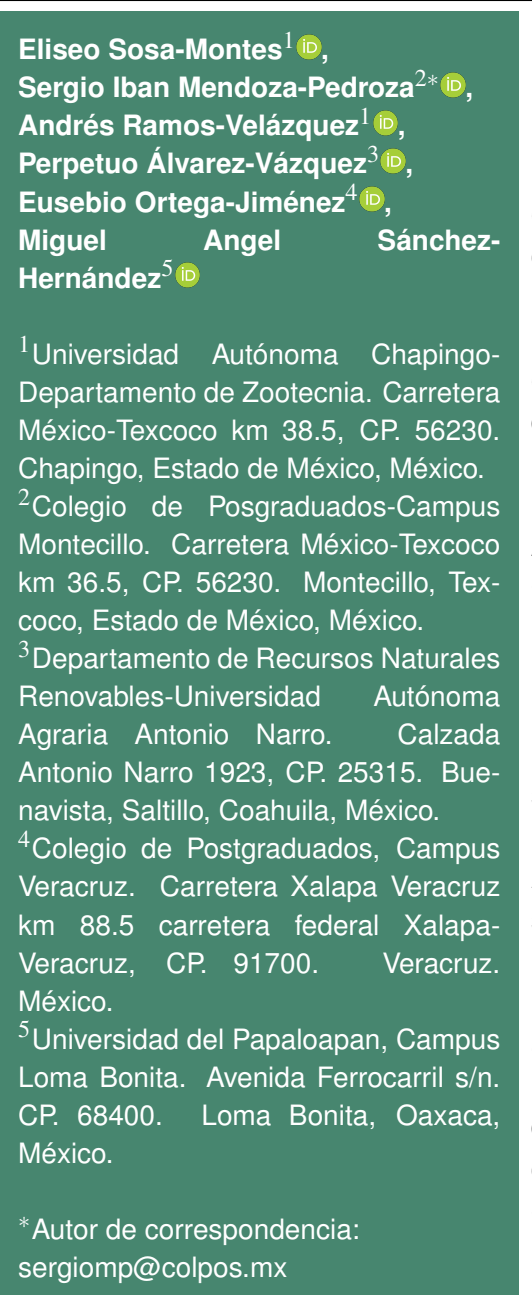

Artículo científico

Recibido: 05 de marzo 2021

Aceptado: 27 de septiembre 2021

Como citar: Sosa-Montes E, Mendoza-Pedroza SI, RamosVelázquez A, Álvarez-Vázquez $P$, Ortega-Jiménez E, SánchezHernández MA (2021) Composición nutricional de tres pastos colectados en el estado de Morelos, México. Ecosistemas y Recursos Agropecuarios Núm. Esp. II: e2968. DOI: 10.19136/era.a8nll.2968
RESUMEN. El objetivo de este estudio fue evaluar tres pastos forrajeros, Bromus diandrus, Oplismenus burmannii y Heteropogon contortus, de las subfamilias Pooideae, Pooideae y Panicoideae, respectivamente. Las principales variables respuesta, todas en base seca fueron: cenizas (Cen), proteína cruda (PC), extracto etéreo $(\mathrm{EE})$, carbohidratos solubles (CS), fibra detergente neutro (FDN), contenido celular (CC = 100 - FDN) y digestibilidad in vitro de la materia seca (DIVMS). Cada pasto se evaluó por triplicado y una repetición se consideró la unidad experimental. Las medias fueron separadas mediante la prueba de Tukey $(p<0.05)$. La asociación entre cada par de variables $(p<0.05)$ se estimó usando el coeficiente de correlación de Pearson $(p<0.05)$. Los pastos $B$. diandrus y $O$. burmannii resultaron con más PC, CC y DIVMS y con menos pared celular o FDN (componente insoluble) que $H$. contortus. Esto produjo una correlación positiva entre CC y PC $(0.751, p<0.05)$. Adicionalmente, $O$. burmannii produjo el valor más alto de Cen y el más bajo de $\mathrm{CS}$, mientras que $B$. diandrus mostró el valor más bajo de Cen y el más alto de CS. Esto produjo una correlación negativa entre estas dos variables $(-0.976, p<0.01)$. En conclusión, $B$. diandrus, mostró mejor composición química y digestibilidad, que los otros dos, por lo que se recomienda este pasto para la nutrición de rumiantes.

Palabras clave: Cenizas, contenido celular, digestibilidad in vitro de la materia seca, proteína cruda.

ABSTRACT. The objective of this study was to evaluate the chemical composition and in vitro digestibility of three forage grasses, Bromus diandrus, Oplismenus burmannii and Heteropogon contortus, subfamilies Pooideae, Pooideae and Panicoideae, respectively. The main response variables, all on dry basis were: ashes (Ash), crude protein (CP), ether extract (EE), soluble carbohydrates (SC), neutral detergent fiber (NDF), cell content $(C C=100-F D N)$ and in vitro dry matter digestibility (IVDMD). Each grass was evaluated in triplicate and one replicate was considered the experimental unit. Means were separated using the Tukey test ( $p$ $<0.05)$. The association between each pair of variables $(p<0.05)$ was estimated by the Pearson correlation coefficient $(p<0.05)$. The grasses $B$. diandrus and $O$. burmannii showed $(p<0.05)$ more CP, CC and IVDMD as well as less cell wall or NDF (insoluble component) than $\mathrm{H}$. contortus. This produced a positive correlation between CC and CP $(0.751 \mathrm{p}<0.05)$. Additionally, $O$. burmannii produced the highest value of Ash and the lowest of SC, while $B$. diandrus showed the lowest value of Ash and the highest of $S C(p<0.05)$. This produced a negative correlation between Ash and SC $(-0.976, p<0.01)$. In conclusion, the grass $B$. diandrus, showed better chemical composition and digestibility than the other two, so this grass is recommended for ruminant nutrition.

Key words: Ashes, cell content, in vitro dry matter digestibility, crude protein. 


\section{INTRODUCCIÓN}

La familia Poaceae incluye aproximadamente 700 géneros y 10000 especies, y México se considera uno de los centros más importantes de diversidad y endemismo de esta familia (DávilaAranda et al. 2004). Los pastos Bromus diandrus, Oplismenus burmannii y Heteropogon contortus pertenecen a la familia Poaceae, subfamilias Pooideae, Panicoideae y Panicoideae, respectivamente (Sánchez-Ken y Cerros-Tlatilpa 2016, Dávila et al. 2018) fueron colectados en Morelos. En este estado se reportaron 104 géneros, 306 especies y ocho subfamilias de la familia Poaceae; y adicionalmente, de las subfamilias mencionadas, las más diversas son: Chloridoideae, Panicoideae y Pooideae (Sánchez-Ken y Cerros-Tlatilpa 2016).

Las plantas de la subfamilia Panicoideae son en su mayoría plantas $\mathrm{C} 4$, mientras que las de la subfamilia Pooideae son C3 (Rommerskirchen et al. 2006). Debido a que los pastos de metabolismo C3 se adaptaron a climas templados, y los de metabolismo C4 lo hicieron a climas tropicales; se habla de dos tipos de pastos: de estación fría y de estación cálida, respectivamente (Bohnert et al. 2011, Norton et al. 2016). Es decir, la temperatura más que la precipitación diferencia entre los pastos C3 y C4 (Edwards y Still 2008). Según datos del INEGI (2021), el estado de Morelos posee una temperatura media anual de $21.5^{\circ} \mathrm{C}$, intermedia entre la del estado de Guerrero $\left(25^{\circ} \mathrm{C}\right)$ de clima tropical y la temperatura de la ciudad de México $\left(16^{\circ} \mathrm{C}\right)$ de clima templado.

De acuerdo con la ecuación de estado de la pared celular (Pietruszka 2020), la cantidad de pared celular y de contenido celular, dependen de la temperatura y del $\mathrm{pH}$, importantes factores del clima y del suelo, respectivamente. La composición química y la digestibilidad entre pastos $\mathrm{C} 3$ y C4, puede diferir; por ejemplo, se considera que la digestibilidad es mayor en pastos C3 que en C4 (Bohnert et al. 2011). Cada una de estas gramíneas puede ser de alto o bajo valor nutritivo (Archimède et al. 2018, Bohnert et al. 2011). Además, no se conoce ampliamente la composición química ni la digestibilidad de los pastos colectados, por tanto, el objetivo del presente trabajo fue evaluar la composición química y la digestibilidad in vitro de la materia seca de tres pastos forrajeros del estado de Morelos, México: Bromus diandrus, Oplismenus burmannii y Heteropogon contortus.

\section{MATERIALES Y MÉTODOS}

\section{Sitios de colecta}

La Tabla 1 muestra los municipios de colecta: Tepoztlán (Heteropogon contortus) y Totolapan (Oplismenus burmannii y Bromus diandrus). Para realizar la identificación botánica y los análisis quími$\cos y$ de digestibilidad in vitro, se obtuvo suficiente cantidad de forraje en tres lugares de cada sitio de colecta (aproximadamente $1000 \mathrm{~g}$ de muestra de cada pasto). Después de un corte de uniformización que se realizó el 15 de septiembre de 2019, se cosechó el forraje a cinco $\mathrm{cm}$ del suelo al inicio de la floración (18 de octubre de 2019). La identificación botánica se realizó en el Herbario del Departamento de Zootecnia, Universidad Autónoma Chapingo (UACh). Después, las muestras se llevaron a peso constante $\left(55^{\circ} \mathrm{C}\right)$ y se realizaron los análisis químicos (AOAC 1990, Van Soest et al. 1991) en el Laboratorio de Nutrición Animal del mismo departamento.

\section{Variables determinadas}

Se determinaron las siguientes variables (AOAC 1990, Van Soest et al. 1991), todas en base seca (\%): cenizas (Cen), proteína cruda (PC), extracto libre de nitrógeno o carbohidratos solubles $(C S)$, extracto etéreo (EE), fibra cruda ( $F C$ ), fibra detergente neutro (FDN) o pared celular, contenido celular $(C C=100-F D N)$, fibra detergente ácido (FDA), lignina (Lig), celulosa (Cel), hemicelulosa (Hcel), sílice (Sí) y digestibilidad in vitro de la materia seca (DIVMS).

\section{Diseño experimental y análisis estadístico}

Se utilizó un diseño experimental completamente al azar con tres tratamientos (los tres pastos forrajeros en estudio) y tres repeticiones, en las cuales se midieron las variables mencionadas. 
Tabla 1. Gramíneas colectadas en el estado de Morelos, México.

\begin{tabular}{|c|c|c|}
\hline Especie & Municipio & Sitio de colecta \\
\hline Bromus diandrus & Totolapan & $\begin{array}{l}\text { Carretera Tlayacapan-Atlatlahuacan, después de la desviación rumbo a Ciudad de México } \\
\left(1711 \mathrm{~m}, 18^{\circ} 58^{\prime} 01^{\prime \prime} \mathrm{N}, 98^{\circ} 58^{\prime} 27^{\prime \prime} \mathrm{O}\right) \text {. }\end{array}$ \\
\hline Oplismenus burmannii & Totolapan & $\begin{array}{l}\text { Antes del puente El Vigía, rumbo a Ciudad de México, en la comunidad Nepopualco (2235 } \\
\left.\mathrm{m}, 19^{\circ} 00^{\prime} 48^{\prime \prime} \mathrm{N}, 98^{\circ} 56^{\prime} 35^{\prime \prime} \mathrm{O}\right) \text {. }\end{array}$ \\
\hline Heteropogon contortus & Tepoztlán & $\begin{array}{l}\text { Paraje El Puente, en la comunidad de Santa Catarina, Tepoztlán ( } 1645 \mathrm{~m}, 18^{\circ} 58^{\prime} 21^{\prime \prime} \mathrm{N} \text {, } \\
\left.99^{\circ} 07^{\prime} 42^{\prime \prime} \mathrm{O}\right) .\end{array}$ \\
\hline
\end{tabular}

Después de realizar el análisis de varianza, se utilizó la prueba de Tukey para la comparación de medias $(p<0.05)$ y se asociaron las variables por pares usando la correlación de Pearson $(p<0.05)$. Para estos análisis se empleó el paquete estadístico para ciencias sociales (SPSS), Statistical Package for the Social Sciences por sus siglas en inglés (SPSS 2011) versión 8.0.

\section{RESULTADOS}

\section{Análisis proximal, digestibilidad in vitro y análisis de Van Soest}

La variable EE no fue diferente entre pastos ( $p$ $>0.05$ ). El pasto Bromus diandrus produjo el menor contenido de Cen, valores intermedios de PC y FC, y el valor más alto de CS (Tabla $1, p<0.05$ ). Oplismenus burmannii produjo los valores más altos de Cen y PC, y los más bajos de CS y FC (Tabla 2, $p$ $<0.05)$. Heteropogon contortus produjo el valor más alto de FC, el más bajo de PC, y valores intermedios de CS y Cen.

Por tanto, B. diandrus mostró los mejores valores del Análisis Proximal, es decir resultó baja en Cen y FC e intermedia en PC, pero no muy lejos del valor más alto de proteína que correspondió a $O$. burmannii. El pasto $B$. diandrus fue seguido por $O$. burmannii en cuanto a los mejores valores de las variables del Análisis proximal.

Las variables FDA, Lig, Cel y Hcel no difirieron $(p>0.05)$ entre los pastos estudiados. $B$. diandrus mostró los valores más altos de CC y DIVMS, y el valor más bajo de FDN o pared celular $(p<0.05$, Tabla 3). O. burmannii también produjo los valores más altos de DIVMS y fue intermedio en las demás variables de Van Soest. (Tabla 2, $\mathrm{p}<0.05$ ). H. contortus produjo el valor más alto de FDN, y los más bajos de CC y DIVMS.

En comparación con los otros dos pastos, $B$. diandrus mostró los mejores valores del Análisis de Van Soest, es decir resultó con baja pared celular (FDN) y fue alto en CS y DIVMS. B. diandrus fue seguido por $O$. burmannii en cuanto a los mejores valores de las variables de Van Soest.

\section{Correlaciones}

En la Tabla 4 se muestran los coeficientes de correlación significativos $(p<0.05$ y $p<0.01)$ entre pares de variables, el coeficiente entre $C C$ y $P C$ fue positivo, el coeficiente entre EE y Hcel fue negativo y entre EE y PC fue positivo. Contrariamente, el coeficiente entre FDN y Hcel fue positivo y entre FDN y PC fue negativo. El coeficiente entre Sí y CS fue negativo y entre Sí y Cen fue positivo. El coeficiente entre Hcel y PC fue negativo y también lo fue entre CS y Cen.

En general, los componentes insolubles (hemicelulosa, cenizas y sílice) mostraron correlaciones negativas $(p<0.05)$ con los solubles (extracto etéreo, contenido celular, carbohidratos solubles y proteína cruda). Los componentes solubles, entre sí, resultaron positivamente correlacionados, y así resultaron los componentes insolubles $(p<0.05)$.

\section{DISCUSIÓN}

\section{Análisis proximal, digestibilidad in vitro y análisis de Van Soest}

Para Bromus diandrus se han reportado valores promedio de PC, CC, FDA y DIVMS de 12.8, 40.9, 31.8 y $64.4 \%$, respectivamente (Hubbard et al. 2011). Para Oplismenus burmannii se reportaron valores de Cen (6\%), PC (7.5 a 8.4\%), CC (26.2 a 35.6), FDA (36.2 a 41.7), Lig (8.3 a 10.0), Cel (25.7 a 27.1), Hcel (27.2 a 30.2) y DIVMS (47.6\%) (Adjorlolo 
Tabla 2. Análisis proximal de tres gramíneas del estado de Morelos, México.

\begin{tabular}{lccccc}
\hline Gramínea & Cen & PC & CS & EE & FC \\
\hline Bromus diandrus & $9.30^{c}$ & $9.37^{b}$ & $46.35^{a}$ & 2.57 & $33.27^{b}$ \\
Oplismenus burmannii & $23.22^{a}$ & $10.23^{a}$ & $37.27^{c}$ & 2.46 & $27.63^{c}$ \\
Heteropogon contortus & $14.71^{b}$ & $5.20^{c}$ & $44.05^{b}$ & 1.47 & $34.98^{a}$ \\
\hline$a, b, c$ & Letras diferentes en la misma columna indican diferencias estadísticas (p $^{\text {< }}$ 0.05), ${ }^{a}$ : valores altos, ${ }^{b}$ : valores intermedios, ${ }^{c}$ : valores bajos. Cen: Cenizas, \\
PC: proteína cruda, CS: carbohidratos solubles, EE: extracto etéreo, FC: fibra \\
cruda.
\end{tabular}

Tabla 3. Digestibilidad in vitro y análisis de Van Soest de tres gramíneas del estado de Morelos, México.

\begin{tabular}{lcccccccc}
\hline Gramínea & DIVMS & FDN & CC & FDA & Lig & Cel & Hcel & Sí \\
\hline Bromus diandrus & $79.03^{a}$ & $70.37^{c}$ & $29.63^{a}$ & 46.43 & 3.72 & 33.57 & 26.69 & 0.77 \\
Oplismenus burmannii & $72.39^{a b}$ & $77.63^{b}$ & $22.37^{b}$ & 51.41 & 4.29 & 29.89 & 26.69 & 9.32 \\
Heteropogon contortus & $62.71^{b}$ & $84.69^{a}$ & $15.31^{c}$ & 53.77 & 7.06 & 34.58 & 30.93 & 6.56 \\
\hline$a, b, c$ & Letras diferentes en la misma columna indican diferencias estadísticas & $(\mathrm{p}<0.05),{ }^{a}$ : valores altos, \\
${ }^{b}$ : valores intermedios, ${ }^{c}$ : valores bajos. DIVMS: digestibilidad in vitro de la materia seca, FDN: fibra \\
detergente neutro o pared celular, CC: contenido celular, FDA: fibra detergente ácido, Lig: lignina, Cel: \\
celulosa, Hcel: hemicelulosa. Sí: sílice.
\end{tabular}

et al. 2014, Geng et al. 2020). Para Heteropogon contortus, se han reportado valores de Cen, PC, CC, FDA, Lig, Hcel y DIVMS de 6.4 a $7.5,3.8$ a $6.6,16.9$ a $48.0,22.0$ a $52.8,3.7,30.0$ y 44.2 a $48.6 \%$, respectivamente (Aganga et al. 2005, Sultan et al. 2008).

Tabla 4. Correlaciones significativas (Pearson, $p<$ 0.05 y $\mathrm{p}<0.01$ ) entre pares de variables respuesta, de tres gramíneas del estado de Morelos, México.

\begin{tabular}{ccccc}
\hline & Hcel & CS & PC & Cen \\
\hline EE & $-0.856^{\star}$ & $0.912^{*}$ & & \\
CC & $-0.748^{*}$ & $0.751^{*}$ & & \\
Sí & & $-0.871^{\star *}$ & & $0.937^{\star \star}$ \\
Hcel & 1 & & $-0.812^{\star *}$ & \\
CS & & 1 & & $-0.976^{\star \star}$ \\
Cen & & & & 1 \\
\hline
\end{tabular}

EE: extracto etéreo, CC: contenido celular, Sí: sílice, Hcel: hemicelulosa, CS: carbohidratos solubles, Cen: cenizas, PC: proteína cruda. ${ }^{*} \mathrm{p}<0.05,{ }^{* *} \mathrm{p}<0.01$.

De entre 11 a 13 datos por variable consultados en la literatura, se encontró en promedio al compararlos con $H$. contortus, que $B$. diandrus y $O$. burmannii resultaron con más PC, CC y DIVMS, es decir, alto valor de CC indica alto valor de PC y alto de DIVMS; estos dos pastos también mostraron valores bajos de pared celular o FDN (componente insoluble).

Los altos valores de PC, CC y DIVMS y bajos de FDN de $B$. diandrus reportados en las referencias citadas al inicio de esta discusión, son congruentes con los encontrados en el presente trabajo (Tabla 2 y
Tabla 3). Los altos valores de pared celular encontrados en $\mathrm{H}$. contortus probablemente se deben a su metabolismo $\mathrm{C} 4$, y al clima cálido subhúmedo del estado de Morelos (INEGI 2021) en el que esta planta evolucionó y favoreció la síntesis de la pared celular (Pietruszka 2020).

\section{Correlaciones}

Estas relaciones negativas entre FDN con CC y PC, coinciden con los resultados de la Tabla 4 en que se detectó una correlación negativa entre Hcel (contenida en la FDN) y CC $(r=-0.748, p<0.05)$ y una correlación negativa entre Hcel $(r=-0.812, p<$ 0.05 ) y PC (componentes insoluble y soluble, respectivamente), lo cual condujo a una correlación positiva $(r=0.751, p<0.05)$ entre CC y PC (ambos componentes solubles).

Los altos contenidos de PC, CC y DIVMS de $B$. diandrus y $O$. burmannii se explican porque son plantas $\mathrm{C} 3$, mientras que $H$. contortus es una planta C4 de la subfamilia Panicoideae (Ellis et al. 1980, Giraldo-Cañas et al. 2010). Las gramíneas con la vía fotosintética C3 se consideran más nutritivas que las C4 (Barbehenn et al. 2004), y tienen mayor digestibilidad (Bohnert et al. 2011). Por ello, B. diandrus, y O. burmannii ambas de la familia Poaceae, subfamilias Pooideae y Panicoideae, respectivamente, mostraron altos contenidos de pro- 
teína, y los mayores valores de contenido celular y digestibilidad, así como los más bajos valores de paredes celulares, al compararlas con la gramínea $H$. contortus, que es planta C4, de la familia Poaceae, de la subfamilia Panicoideae.

\section{CONCLUSIONES}

Por sus altos contenidos de proteína, con- tenido celular, carbohidratos solubles y digestibilidad, y por sus bajos contenidos de compuestos fibrosos, a la misma edad de corte, se recomienda el uso de $B$. diandrus, en la alimentación de rumiantes. Alternativamente, el uso de $O$. burmannii también se recomienda para rumiantes, debido a sus altos niveles de proteína, contenido celular y digestibilidad, pero teniendo precaución porque esta gramínea acumula un exceso de cenizas.

\section{LITERATURA CITADA}

Adjorlolo L, Adogla-Bessa T, Amaning-Kwarteng K, Ahunu B (2014) Effect of season on the quality of forages selected by sheep in citrus plantations in Ghana. Tropical Grasslands - Forrajes Tropicales 2: 271-277.

Aganga AA, Omphile UJ, Mojaditlhogo N (2005) Composition and digestibility of indigenous grasses in the hardveld of Botswana during the dry season. Archivos de Zootecnia 54: 587-598.

AOAC (1990) Official Methods of Analysis. Association of Official Analytical Chemists. 15th Ed. Arlington, Virginia, USA. 771p.

Archimède $\mathrm{H}$, Rira M, Eugène M, Fleury J, Lastel ML, Periacarpin F, Silou-Etienne T, Morgavi DP, Doreaub M (2018) Intake, total-tract digestibility and methane emissions of Texel and Blackbelly sheep fed C4 and C3 grasses tested simultaneously in a temperate and a tropical area. Journal of cleaner production 185: 455-463.

Barbehenn RV, Chen Z, Karowe DN, Spickard A (2004) $\mathrm{C}_{3}$ grasses have higher nutritional quality than $\mathrm{C}_{4}$ grasses under ambient and elevated atmospheric $\mathrm{CO}_{2}$ Global Change Biology 10: 1565-1575.

Bohnert DW, DelCurto T, Clark AA, Merrill ML, Falck SJ, Harmon DL (2011) Protein supplementation of ruminants consuming low-quality cool- or warm-season forage: differences in intake and digestibility. Journal of Animal Science 89: 3707-3717.

Dávila-Aranda P, Lira-Saade R, Valdés-Reyna J (2004) Endemic species of grasses in Mexico: a phytogeographic approach. Biodiversity and Conservation 13: 1101-1121.

Dávila P, Mejia-Saulés MT, Soriano-Martínez AM, Herrera-Arrieta Y (2018) Conocimiento taxonómico de la familia Poaceae en México. Botanical Sciences 96: 462-514.

Edwards EJ, Still CJ (2008) Climate, phylogeny and the ecological distribution of C4 grasses. Ecology Letters 11: 266-276.

Ellis RP, Vogel JC, Fuls A (1980) Photosynthetic pathways and the geographical distribution of grasses in South West Africa/Namibia. South African Journal of Science 76: 307-314.

Geng Y, Ranjitkar S, Yan Q, He Z, Su B, Gao S, Niu J, Bu D, Xu J (2020) Nutrient value of wild fodder species and the implications for improving the diet of mithun (Bos frontalis) in Dulongjiang area, Yunnan Province, China. Plant Diversity 42: 455-463.

Giraldo-Cañas D (2010) Distribución e invasión de gramíneas C3 y C4 (Poaceae) en un gradiente altitudinal de los Andes de Colombia. Caldasia 32: 65-86.

Hubbard AS, Parish JA, Macoon B, Vann RC, Parish JR (2011) Agronomic performance, plant morphology, and nutritive value among three prairie bromegrass entries. Online. Forage and Grazinglands 9: 1-2. 
INEGI (2021) Instituto Nacional de Estadística, Geografía e Informática. Estados de Morelos, Guerrero y Ciudad de México. http://www.cuentame.inegi.org.mx/monografias/informacion/mor/territorio/clima.aspx?tema=me $\& \mathrm{e}=17$. Fecha de revisión: 13 de enero de 2021.

Norton MR, Malinowski DP, Volaire F (2016) Plant drought survival under climate change and strategies to improve perennial grasses. A review. Agronomy for Sustainable Development 36: 1-15.

Pietruszka MA (2020) Chemical potential-induced wall state transitions in plant cell growth. Journal of Plant Growth Regulation 39: 841-866.

Rommerskirchen F, Plader A, Eglinton G, Chikaraishi Y, Rullkötter J (2006) Chemotaxonomic significance of distribution and stable carbon isotopic composition of long-chain alkanes and alkan-1-ols in C4 grass waxes. Organic Geochemistry 37: 1303-1332.

Sánchez-Ken JG, Cerros-Tlatilpa R (2016) Listado florístico de la familia Poaceae del estado de Morelos, México. Acta Botánica Mexicana 116: 65-105.

SPSS (2011) Statistical Package for the Social Sciences. Institute. SPSS-X. User's Guide. Version 8, Chicago Illinois, USA. www.ibm.com/legal/copytrade.shtml. Fecha de consulta: 13 de enero de 2021.

Sultan JI, Rahim IU, Yaqoob M, Nawaz H, Hameed M (2008) Nutritive value of free rangeland grasses of northern grasslands of Pakistan. Pakistan Journal of Botany 40: 249-258.

Van Soest PJ, Robertson JB, Lewis BA (1991) Methods for dietary fiber, neutral detergent fiber, and nonstarch polysacaccharides in relation to animal nutrition. Journal of Dairy Science 74: 3583-3597. 